\title{
La nozione di ager privatus nella libera res publica e nella costruzione giuridico-istituzionale del circolo scipionico
}

\author{
Osvaldo Sacchi
}

Università degli Studi della Campania Luigi Vanvitelli, Dipartimento di Giurisprudenza Kontaktni e-mail: osvaldo.sacchi@unicampania.it

The Notion of ager privatus in the libera res publica and in the Juridical-Institutional Construction of the Scipionic Circle

\begin{abstract}
:
This article concerns at first the notion of ager privatus qualified as possession (a subtraction from a whole: the ager publicus populi Romani) and not as property as the dominium ex iure Quiritium. The influence of the stoic philosophical thought of the media stoa played a fundamental role in the process of transforming the possession of the ager publicus into a quiritary dominium. Infact, the activity of the participants in the scipionic circle was perhaps a further step in the complex reconstruction of the long and arduous process that sanctioned the transition in Roman law from the possessio of ager publicus to the dominium ex iure Quiritium.
\end{abstract}

Keywords: ager publicus; ager privatus; privatarum possessionum discriptio; Cic. de re p. 1, 25, 39; Cic. de off. 1, 20-21; Cic. de off. 2, 73

DOI: $10.14712 / 2464689 X .2020 .17$

Testo della relazione svolta nel XV Seminario Internazionale di Diritto romano e attualità, Praga, 22 novembre 2019, nell'ambito del progetto di ricerca Società complesse e Governo. Il lascito culturale di Roma antica. Dalla maiestas della civitas alla maiestas del princeps. Sessione dal titolo Consolidameno e sviluppi degli assetti costituzionali nella libera res publica, con aggiunta di note essenziale. 
«Nessun altro schema dell'organizzazione giuridica rivela al pari della proprietà il suo carattere di prodotto storico. (...). La linea storica corre formalmente unitaria ma sostanzialmente scandita in momenti dotati di rilevante specificità» [GROSSI, P. L'inaugurazione della proprietà moderna. Napoli: Guida, 1980, pp . 17-18].

\section{Premessa}

Un anno fa, in occasione del nostro primo incontro di Catanzaro, chiusi la mia relazione in modo alquanto sibillino. Insinuai l'ipotesi del Vico per cui, prima la riforma serviana, e poi le XII tavole, sarebbero state le prime due leggi agrarie della storia di Roma. ${ }^{1}$

Con la prima i plebei avrebbero avuto accesso ad appezzamenti di terreno in regime quasi feudale ottenendo, uno status libertatis consistente nella possibilità di coltivare a giornata le terre dei patrizi (dominio bonitario), ${ }^{2}$ mentre con la seconda, cioè le dodici tavole, questo accesso sarebbe stato riconosciuto come dominio quiritario (heredium/hortus?). ${ }^{3}$

Non è mia intenzione verificare in questa sede l'attendibilità di tale ricostruzione definita da Pasquale Garofalo: «la più aspra delle meditazioni del Vico sulle cose Romane». ${ }^{4}$

1 La Scienza Nuova di Vico del 1744 è citata da qui in poi usando l'ordine dei capoversi e le pagine (indicando col n. 2 solo il secondo volume dell'opera seguito da due punti e l'indicazione di pagina) dell'edizione VICO, G. La Scienza Nuova Seconda giusta l'edizione del 1744 con le varianti dell'edizione del 1730 e di due redazioni intermedie inedite, in due voll. [1: parte prima (libri I-II); 2: parte seconda (libri III-V e Appendice)]. NICOLINI, F. (a cura di). Bari: Gius. Laterza \& Figli, 1953; [= VICO, G. La Scienza Nuova 1744: testo, manoscritto, editio princeps. Edizione elettronica a cura del Centro di Umanistica Digitale dell'ISPF-CNR. Laboratorio dell'ISPF. 2015, Vol. XII. DOI: 10.12862/ispf15L101. [online]. [30. 12. 2015], da cui traggo le citazioni testuali (d'ora in poi citata come web con la pagina corrispondente)]; [= Principj di Scienza Nuova di Giambattista Vico d'intorno alla comune natura delle nazioni, rist. anast. DE GIOVANNI, B. (a cura di). Napoli: Belle Époque Edizioni, 2019, (da qui in poi citata come anast. con la pagina corrispondente)].

2 Vico SNS 1744 cpv. 107, p. 66 (56 web; 66 anast.): «Appresso Servio Tullio vi ordinò il Censo, con permettere a' giornalieri il dominio bonitario de' campi, ch'erano propj de' Padri; i quali essi coltivassero per sé, sotto il peso del censo, con l'obbligo di servir loro a propie spese nelle guerre; conforme di fatto i plebei ad essi Patrizj servirono dentro cotesta finor sognata libertà popolare: la qual Legge di Servio Tullio fu la prima Legge Agraria del Mondo, ordinatrice del Censo pianta delle Repubbliche Eroiche, ovvero antichissime Aristocratie di tutte le Nazioni».

3 Vico SNS 1744 cpv. 109, p. 67 (56 web; 66-67 anast.): «Per tutto ciò pur seguitando i Nobili a ritogliere i campi a' plebei, poichè quelli gli avevano coltivati; nè avendo questi azion civile da vendicargli, quivi i Tribuni della plebe fecero la pretensione della Legge delle XII. Tavole (dalla quale, come ne' Principj del Diritto universale si è dimostrato, non si dispose altro affare, che questo) con la qual legge $i$ Nobili permisero il dominio quiritario de' campi a' plebei; il qual dominio civile per diritto natural delle genti permettesi agli stranieri: e questa fu la seconda legge Agraria dell'Antiche Nazioni».

4 GAROFALO, P. Acrisia Vichiana nella "Scienza Nuova". Annotazioni critiche. Napoli: Enrico Denkten, 1909, pp. 5-539, spec. p. 332. La curiosa Acrisia Vichiana, a firma di un signor Pasquale Garofalo di Bonito, secondo Nicolini (NICOLINI, F. Commento storico alla Seconda Scienza Nuova, I. Roma: Edizioni di Storia della Letteratura, 1949, pp. 14-15) sarebbe un brogliaccio attribuito a Nicola Corcia, il grande antichista napoletano in gioventù fervente vichiano, mentre in vecchiaia altrettanto fervente antivichiano. Proprio su questo punto assai ostico della storia di Roma, il Garofalo/(Corcia?) s'impegna molto per rilevare quanto il Nostro si discosti dalla trama letterale degli storici antichi, senza però scalfire (a mio avviso) la carica suggestiva della ricostruzione vichiana. Questa prima seria lettura critica della tradizione del Vico può tornare utile ai romanisti che, più di altri, sono in grado di cogliere la parte nascosta del pensiero di questo grande napoletano. Il Nicolini definisce la ricostruzione vichiana «tra le maggiori scoperte storiche del Vico» (Commento storico, I, ivi 67) e con termini ancora più coloriti prosegue (ibidem): «più che in ciascuna considerata in sé e per sé, la loro importanza grande è nel loro complesso e ancora più nell'orientamento, del tutto precorritore, a cui Vico s'ispirò nel formularle: il che consente bene di parlare di un colpo di catapulta, il quale, mentre sfasciava quello scenario di carta dipinta che 
Devo confessare però che non avevo mai considerato, prima di leggere il Vico, che si potessero configurare l'ordinamento serviano e le XII tavole come le prime due leggi agrarie della storia di Roma. Così come non avevo saputo finora apprezzare fino a che punto, le nozioni di res publica e di "proprietà" enunciate nel circolo scipionico (un dibattito che portò probabilmente alla prima forma di costituzionalismo consapevole del diritto romano), potrebbero essere state condizionate dalla crisi agraria dell'età graccana. Lo stesso direi per l'affermazione del concetto di "proprietà" quiritaria (in età classica definito dominium ex iure Quiritium che Gaio parlando del duplex dominium affianca all' in bonis habere pretorio):

Gai 1, 54: Ceterum cum apud cives Romanos duplex sit dominium (nam vel in bonis vel ex iure Quiritium vel ex utroque iure cuiusque servus esse intellegitur), ita demum servum in potestate domini esse dicemus, si in bonis eius sit, etiamsi simul ex iure Quiritum eiusdem non sit: Nam qui nudum ius Quiritium in servo habet, is potestatem habere non intellegitur.

Gai 2, 40: Sequitur, ut admoneamus apud peregrinos quidem unum esse dominium; nam aut dominus quisque est aut dominus non intellegitur. quo iure etiam populus Romanus olim utebatur: aut enim ex iure Quiritium unusquisque dominus erat aut non intellegebatur dominus. sed postea diuisionem accepit dominium, ut alius possit esse ex iure Quiritium dominus, alius in bonis habere.

Per l'età più antica si deve però pensare a una tipologia di appartenenza "unitaria" che prima della scissione in duplum non può che essere stata il meum esse potestativo del pater: si vedano ad esempio le personae in mancipio di Gaio $(1,116)$, le res pretiosiores $(1,192)$, le più antiche servitù rustiche e forse anche l'heredium. ${ }^{5}$

ai tempi del Nostro era ancora la storia di Roma primitiva, la poneva, al tempo stesso, per la strada così diversa che avrebbe percorso per tutto il secolo XIX».

5 Cfr. GIUFFRÈ, V. Beni della vita e diritto. In: Il bisogno del diritto. Momenti dell'esperienza romanistica. Napoli: Jovene, 2007, p. 27 ss. Il problema della natura giuridica dell'originaria signoria del pater familias è arduo e non ha trovato ancora consenso unanime in dottrina. Per la teoria potestativa v. FRANCIOSI, G. Usucapio pro herede. Contributo allo studio dell'antica hereditas. Napoli: Jovene, 1965, p. 31 ss.; Idem. Famiglia e persone in Roma antica. Dall'età arcaica al principato. Torino: Giappichelli, 1995, pp. 43-47, spec. 46. Contra SANTORO, R. Potere ed azione nell'antico diritto romano. In: Annali del Seminario Giuridico della Università di Palermo, XXX. Palermo, 1967, p. 114 e passim. Cfr. anche GALLO, F. Osservazioni sulla signoria del pater familias in epoca arcaica. In: Studi De Francisci, 2. Milano: Giuffrè, 1956, p. 193 ss.; Idem. 'Potestas' e ‘dominium' nell'esperienza giuridica romana. Labeo, 1970, 16, p. 17 ss.; CAPOGROSSI COLOGNESI, L. La struttura della proprietà e la formazione dei iura praediorum in età repubblicana, 1. Milano: Giuffré, 1969, p. 105 ss.; Idem. Ancora sui poteri del 'pater familias'. Bullettino dell'Istituto di Diritto Romano, 1970, 73, p. 357 ss.; CORBINO, A. Schemi giuridici dell'appartenenza nell'esperienza romana arcaica. In: Scritti Falzea. Napoli, 1987, p. 43 ss. Mi pare però significativo quanto scrive con riferimento all'ambito di applicazione della legis actio sacramenti in rem SOLIDORO MARUOTTI, L. Riflessioni sulla struttura della rivendica romana e sulla prova della proprietà. In: D'IPPOLITO, F. M. (a cura di). Scritti in onore di G. Franciosi. Napoli: Satura, 2007, pp. 2473-2506 [1-34], spec. p. 2488 [16]: «Si può ormai ritenere sufficientemente dimostrato che nell'età arcaica non si era ancora tracciata una netta linea di demarcazione tra le forme possessorie, il diritto di proprietà, le potestà familiari, i diritti reali limitati: il potere unitario del pater (mancipium, o ius) si articolava al suo interno in una estrema varietà di schemi giuridici in ragione dei numerosi oggetti (schiavi, figli, donne, terre, animali, strumenti di lavoro, ecc.), mentre in termini di concettualizzazione giuridica, corrispondeva a una generica 'appartenenza' di res e persone». A mio avviso questa lettura corrisponde esattamente alla visione che può aver avuto il giurista Gaio che scrive su questi temi nel II secolo d.C. quando la distinzione di matrice retorica tra res e personae è diventata ormai consueta per il lessico giuridico romano. Lo dimostra fra l'altro quanto scrive il giurista stabilendo una stretta connessione tra l'effettivo godimento del bene giuridico (in questo caso il servus) e lo ius potestatis: Gai 1, 54: ita demum servum in potestate 
Il dibattito sulla proprietà della terra di cui discussero i membri del circolo scipionico può allora aver influito in maniera determinante non solo (indirettamente) sulla mutazione del meum esse originario in dominium; ma anche (più direttamente) sulla trasformazione del modo d'intendere le varie tipologie di ager privatus della lex agraria del 111 a.C. ${ }^{6}$ Da situazioni possessorie qualificate, queste cominciarono a configurarsi come espressioni di proprietà quiritaria (o ex iure Quiritium) in senso stretto forse anche grazie a questo dibattito. ${ }^{7}$

Mi è parso quindi che, nel quadro di una disamina generale sul consolidamento e lo sviluppo degli assetti costituzionali nella libera res publica, forse valesse la pena di attirare l'attenzione su questo momento molto particolare della storia di Roma, quando la cultura ellenistica trasformò il diritto romano in un'esperienza universale.

\section{La nozione di ager privatus e la lex agraria del 111 a.C.}

Per dare ordine all'esposizione comincerei brevemente dalla figura dell'ager privatus. Rispetto a lucuzioni generiche come ager gentilicius, ager vacuus, ager publicus o tecnico-sacrali più specifiche come nel diritto augurale (Varro l.L. 5, 5, 33: Ut nostri augures publici disserunt, agrorum sunt genera quinque: Romanus, Gabinus, peregrinus, hosticus, incertus), una diversa concezione più sofisticata di ager qualificato come privatus, fa la sua comparsa nella legge agraria epigrafica del 111 a.C., dedicata alle terre e ai possedimenti ager publicus populi Romani localizzati in territorio italiano e oltre penisola, che è la fonte principale sull'organizzazione fondiaria romana e sulle tipologie di possesso della terra a Roma per l'epoca immediatamente postgraccana. Evito di riprodurre qui un elenco che sarebbe molto articolato e mi limito, solo a titolo di esempio, a riportare le prime parole leggibili della linea 8 relative ai lotti di terreno in Italia (ager datus adsignatus):

linea 8: utei ceterorum locorum agrorum aedificiorum privatorum est, esto.

Questa qualificazione dell'ager datus adsignatus configurato come ager privatus era nota a Catone, ma già Plauto impiega il vocabolo privatus nel senso di "singolo" o di semplice di "cittadino", in contrapposizione a ciò che invece esprimeva una carica pubblica. Il dato trova conferma anche in Cicerone, Tacito e nel tardo Isidoro. ${ }^{8}$

domini esse dicemus, si in bonis eius sit, etiamsi simul ex iure Quiritum eiusdem non sit: Nam qui nudum ius Quiritium in servo habet, is potestatem habere non intellegitur.

6 Mi permetto di rinviare per questo a SACCHI, O. Regime della terra e imposizione fondiaria nell'età dei Gracchi. Testo e commento storico-giuridico della legge agraria del 111 a.C. Napoli: Jovene, 2006, pp. XXIII-627, spec. p. 59 ss. Ma v. ora CHOUQUER, G. Les catégories de droit agraire à la fin du IIe s. av.J.-C. (senentia Minuciorum de 117 av.J.-C.et lex agraria de 111 av.J.-C.). Paris: Ouvrage édité par L'Observatoire des formes du foncier dans le monde, 2016, p. 58 ss., 152-153 e passim.

7 Ben inquadra l'emersione della figura del dominium ex iure Quiritium SOLIDORO MARUOTTI, L. La tradizione romanistica nel diritto europeo. II. Dallo ius commune alle codificazioni moderne. Torino: Giappichelli, 2003, p. 242: «La nuova figura del dominium ex iure Quiritium, pur avendo contenuto economico e connotandosi, quindi, come un diritto patrimoniale, si andava a innestare nella antica concezione pre-etrusca della 'sovranità' del pater o forse, (secondo una differente impostazione), della sua 'potestà' (Franciosi), da intendersi in ogni caso come un tipo di potere 'solitario', in quanto 'estraneo' e indifferente ai rapporti giuridici con gli altri consociati (Candian). Questo 'innervamento' del dominium nella antica concezione potestativa - o addirittura sovrana - fu reso possibile dallo spirito individualistico (da alcuni definito addirittura 'liberale') che indubbiamente pervase, in qualche misura, l'età repubblicana. È appunto sulla base di questi elementi che la figura del dominium ex iure Quiritium è stata poi, nei secoli successivi, costantemente evocata per costruire il modello di elezione di una immagine individualistica e liberale (Locke), non sociale della proprietà».

8 Cato orat. 79 fragm. 206: Accessit ager, quem privatim habent, Gallicus, Samnitis, Apulus, Bruttius; Plaut. Capt. 166: hic qualis imperator nunc privatus est; Cic. de leg. 3, 19, 43: Deinde de promulgatione, de singulis rebus agendis, de privatis magistratibusve auduendis; Phil. 11, 25: Valde mihi probari potest, 
Per chiarire cosa debba intendersi per privatus è molto indicativa però una nota testimonianza di Festo tratta dall'epitome di Paolo da leggere insieme a Plauto (Poen. 775: ut eo me privent atque inter se dividant) e Cesare (b.civ. 3, 90, 3: rem publicam alterutro exercitu privare):

Paul.-Fest. sv. Privos privasque (L. 252, 20): Privos privasque antiqui dicebant pro singulis. Ob quam causam et privata dicuntur, quae uniuscuiusque sint; hinc et privilegium et privatus; dicimus tamen et privatum, cui quid est ademptum.

Qui si vede bene che privatus è una parola dal significato non univoco: da un lato indica ciò che è del singolo (privos privasque antiqui dicebant pro singulis) come in Gai 2, 11: Privatae [res] sunt quae singulorum hominum sunt; dall'altro ciò che è "sottratto", ossia ademptum (dicimus tamen et privatum, cui quid est ademptum); come due facce della stessa medaglia. Questa ambivalenza è coerente con una certa ambiguità che sembra essere stato il tratto caratteristico della disciplina giuridica fondiaria romana lungo tutto il corso dell'età repubblicana. Si pensi all'heredium rispetto alla natura collettiva dell'originaria "proprietà quiritaria"; 9 ovvero all'idea che la proprietà privata non esista in natura sebbene il compito della res publica fosse quello di proteggere ciò che è di ciascuno. ${ }^{10}$ Lo stesso si può dire del modus agri che ancora in età classica appare un'astratta misura di terra inscritta in una forma o griglia centuriale senza indicazione dei confini, ma alienabile mediante mancipatio. ${ }^{11}$

Ad ogni modo, per un valore semantico di privatus nel significato più chiaro di "proprietà privata", in un senso anche patrimoniale, dobbiamo attendere almeno fonti del principato. ${ }^{12} \mathrm{E}$ forse risale ad età augustea, come segnalò il Mommsen, anche la prima attestazione di proprietas che è presente nell'Elogio di Murdia: ${ }^{13}$

CIL VI 10230: ut ea ussu suo custodia proprietati meae restituerentur.

qui paulo ante clarissimo viro privato imperium extra ordinem non dedi; Tac. agr. 39, 2: Id sibi maxime formidolosum, privati hominis nomen supra principem attolli; Isid. etym. 9, 4, 30: Privati sunt exstranei ab officiis publicis. Est enim nomen magistratum habenti contrarium, et dict privati quod sint ab officiis curiae absoluti.

9 Cfr. CAPOGROSSI COLOGNESI, L. Ager publicus e ager gentilicius nella riflessione storiografica moderna. In: Studi in onore di Cesare Sanfilippo, III. Milano: Giuffrè, 1983, p. 73 ss. (= Scritti scelti, I. Napoli: Jovene, 2010, p. 655 ss.) e ora, per il sofferto percorso storiografico sul tema, si v. FRANCIOSI, A. Alcune riflessioni in tema di heredium e terre gentilizie. In: Scritti per Alessandro Corbino, 3. Tricase (LE): Libellula, 2016, pp. 155-171.

10 Cic. de off. 1, 20-21 per cui v. infra.

11 Gai 1, 120; 2, 29; D. 11, 6 (Si mensor falsum modum dixerit); D. 10, 1, 7 (Modest. 11 pand.); Front. de contr. agr. 13, 7-14, 7 (Lach.). Sul modus agri e il suo inquadramento storico-giuridico mi sia permesso rinviare a SACCHI, O. Ager est, non terra. Dall'ager privatus alla forma agrimensorum: evoluzione di un paradigma tra natura, diritto, anomalismo e analogismo giuridico. In: Questioni della terra. Società, economia, normazioni, prassi in onore di Mariagrazia Bianchini. Atti dell'Accademia Romanistica Costantiniana XXII. Napoli: Edizioni Scientifiche Italiane, 2017, p. 178, n. 45. E ancora CHOUQUER, op. cit., pp. 55-57 e passim.

12 Ovid.fast. 5, 286: vindice servabat nullo sua publica volgus,/iamque in privato pascere inertis erat; Liv. 2 , 24, 7: ex tota urbe proripientium se ex privato; Liv. 30, 44, 11: nunc quia tributum ex privato conferendum est; Sen. epist. 89, 20: Inlustrium fluminum per privatum decursus est et amnes magni magnarumque gentium termini usque ad ostium a fonte vestri sunt.

13 Così MOMMSEN, Th. Mancipium. Manceps. Praes. Praedium. ZSS, 1902, 23, p. 439, n. 1. Si v. anche SACCHI, Ager est, non terra, p. 169 e n. 21. 


\section{L'emersione di una concezione mercantile della proprietà fondiaria}

Questa configurazione giuridica dell'ager privatus come una sottrazione di ager publicus (da ademptum participio di adīmo, adīmere = "strappare", "togliere") - che si affianca alla preesistente nozione augurale di ager - aprì dunque la strada anche alla vicenda dell'emersione della categoria giuridica del dominium (quiritario o ex iure Quiritium) dove, l'appartenenza fondiaria, si connota in un senso eminentemente patrimoniale.

Sappiamo che la sostituzione di erus con dominus avvenne solo nel De agri cultura di Catone (II sec. a.C.) (Capogrossi) e le ragioni che portarono alla mutazione di erus in dominus sul piano dell'evoluzione socio-economica dovrebbero essere evidenti: da una concezione "naturale" di erus come legittimo "signore" dello spazio (in primis l'heredium insieme all'hortus) che gli competeva per vivere insieme al suo nucleo familiare - pur all'interno di una dimensione collettiva dell'originaria appartenenza o "proprietà quiritaria" (gentilizia o consortium ercto non cito) si è passati col tempo a una concezione "artificiale", meramente giuridica, di dominus come "signore" nel senso di chi esercita un dominio per averlo acquisito a vario titolo legalmente riconosciuto. ${ }^{14}$ A Roma si cominciò con la predazione bellica, poi, via via, secondo una tassonomia che, come vedremo più avanti, Cicerone espliciterà con la disinvoltura di chi sa di cosa parla. ${ }^{15} \mathrm{Su}$ questa tassonomia, a partire da Panezio, è possibile però che abbiano ragionato anche i componenti del circolo scipionico che furono protagonisti e testimoni dei tumulti graccani.

\section{I personaggi del circolo scipionico}

Arriviamo così al cuore di questo mio articolo. Come ho già ribadito anche in altre occasioni, una indagatissima testimonianza del De re publica $(1,25,39)$ e altre due altrettanto celebri testimonianze del De officiis $(1,20-21$ e 2,73$)$ potrebbero fornire la testimonianza di un vero momento di svolta, non solo per la storia del costituzionalismo antico, ma anche per l'evoluzione dell'idea di appartenenza fondiaria nel diritto romano tardo repubblicano e classico. Comincio con qualche considerazione sul circolo scipionico.

Intorno a Publio Scipione Emiliano Africano Minore Numantino (nato alla fine del 185 o all'inizio del 184 a.C.), il distruttore di Cartagine, e al suo fraterno amico Gaio Lelio Sapiente (nato nel 190 circa a.C.) è abbastanza noto che si aggregò un gruppo di uomini (definito graex Scipionis: Cic. Lael. 69) che condivise in linea di massima le stesse tendenze politiche.

Lelio fu di origini plebee e autore nel 145, quando pretore, di un progetto di riforma agraria di ripartizione dell'ager publicus (Plut. Tib. Gracc. 8, 3), poi ritirato; cosa che gli

14 Si v. per questo CAPOGROSSI COLOGNESI, La struttura della proprietà e la formazione dei iura praediorum in età repubblicana, p. $442 \mathrm{ss}$. Ma v. anche di tale studioso La proprietà in Roma dalla fine del sistema patriarcale alla fioritura dell'ordinamento schiavistico. In: La terra in Roma antica. Forme di proprietà e rapporti produttivi. I (Età arcaica). Roma: La Sapienza, 1981, p. 135 ss. Siamo forse ancora di fronte a una manifestazione della capacità potestativa del pater familias e non quindi già alla qualificazione giuridica di una relazione proprietaria/patrimoniale tra un soggetto e una res (la particella di terreno viritana). Sul tema molto discusso v. SOLIDORO MARUOTTI, L. 'Proprietà assoluta' e 'proprietà relativa' nella storia giuridica europea. Drevnee pravo/Ius Antiquum, 2004, 14, 2, Mosca, pp. 7-50, spec. 17 , dove sottolinea la mancanza ancora nel II secolo a.C. di vocaboli atti a esprimere un'idea astratta della signoria giuridica su una cosa, cioè un'idea astratta di proprietà. 
valse l'appellativo di бoøó (Plut. Tib. Gracc. 8, 5). ${ }^{16}$ Da parte sua, Scipione Emiliano, si impegnò per togliere facoltà decisionale ai triumviri agris adsignandis iudicandis di Tiberio Gracco (suo cognato e cugino: Plut. Tib. Gracc. 7, 4), che volevano espropriare gli italici dei loro possedimenti privati di ager publicus legittimati da foedera. Col trasferimento di tale giurisdizione al console Tutidano, la lex Semponia agraria fu così grazie a Scipione di fatto resa ineffettiva. ${ }^{17}$ Sembra quindi credibile Cicerone anche quando afferma che Scipione approvò la morte di Tiberio. ${ }^{18}$ Insomma, si può dire che i due personaggi romani politicamente più autorevoli del sodalizio scipionico, rispetto alla crisi graccana, sostennero una politica di consolidamento dello status quo e di ferma opposizione a ogni tentativo di riforma democratica. Fu in questo clima però che Scipione, Panezio, Polibio, Lelio e molti altri ancora, discussero anche sulle ragioni della grandezza di Roma al momento in cui evidentemente si ebbe per la prima volta piena consapevolezza della sua potenza. Di questo siamo sicuri perché questa convinzione era diffusa anche tra gli storici antichi: Vell. Pat. 2, 1, 1: Potentiae Romanorum prior Scipio viam aperuerat, luxuriae posterior aperuit.

\section{La definizione «res publica, res populi» in de re p. 1, 25, 39}

Veniamo allora alla testimonianza del De re publica di cui si diceva prima:

Cic. de re p. 1, 25, 39: «Est igitur», inquit Africanus, «res publica res populi, populus autem non omnis hominum coetus quoquo modo congregatus, sed coetus multitudinis iuris consensu et utilitatis communione sociatus». eius autem prima causa coeundi est non tam inbecillitas quam naturalis quaedam hominum quasi congregatio.

Da questo luogo si comprende che idea di "Stato" intesero i protagonisti del sodalizio scipionico. Cicerone, come è noto, immagina un dialogo iniziato il mattino del primo giorno delle feriae latinae negli horti suburbani dell'Emiliano e sceglie come data il 129 a.C. perché fu un anno denso di avvenimenti drammatici: apparvero nel cielo due soli; ${ }^{19}$ vi furono gravi moti sociali; e Scipione, appena 56enne, dopo trascorse le ferie latine, morì in circostanze mai del tutto chiarite. ${ }^{20}$ Tutti i personaggi del De re publica (oltre l'Emiliano, Lelio, Spurio Mummio, Elio Tuberone, Rutilio Rufo, Mucio Scevola Augure e Fannio) furono inoltre sodales del circolo scipionico tranne Manilio, forse incluso perché generale di Scipione a Cartagine e/o per dare maggiore spessore giuridico alle argomentazioni dei personaggi. ${ }^{21}$

16 ROTONDI, G. Leges publicae populi Romani (1912). Hildesheim - Zürich - New York: Olms, 1990, p. 294; CANCELLI, F. Introduzione a Marco Tullio Cicerone, Lo Stato. CANCELLI, F. (a cura di). Milano: Mondadori, 1979, p. 49.

17 App. b.civ. 1, 19, 78-80: «Gli Italici, non sopportando questa situazione e la rapidità con la quale i triumviri giudicavano in questa materia, pregavano Cornelio Scipione, quello che aveva distrutto Cartagine, di farsi loro patrono contro le ingiustizie. [79] Egli, che li aveva conosciuti valorosissimi in guerra, non osò trascurarli e, recatosi al Senato, non biasimò apertamente la legge di Gracco, per non disgustare il popolo, ma, esposte le difficoltà che ne derivavano, espresse il parere che le controversie non fossero decise dai triumviri, sospetti ai giudicandi, ma da altri. [80] Con questa proposta, che appariva giusta, riuscì facilmente a convincere; e il console Tutidano fu incaricato della funzione giudicatrice. Ma egli, accintosi all'impresa e vistane la difficoltà, intraprese una campagna contro gli Illiri, per aver così un pretesto per non occuparsi dei giudizi. I triumviri agrari, poiché nessuno si presentava loro per i giudizi, riamsero inattivi». Trad. it. di GABBA, E. (a cura di). La storia romana. Le guerre civili di Appiano. Torino: Utet, 2001, p. 83.

18 Cic. de orat. 2, 106; de dom. 91; pro Planc. 88; de off. 1, 76; 2, 43; Vell. Pat. 2, 4, 4.

19 Fenomeno ben noto ai romani: Liv. 28, 11, 3; Plin. NH 2, (31), 99 e Sen. nat. 1, 11, 2.

20 Sulla morte di Scipione cfr. CANCELLI, Introduzione, pp. 43-44.

21 Per i dettagli v. CANCELLI, Introduzione, pp. 38-56. 
$\grave{E}$ risaputo che gran parte di questo dialogo ruota intorno alla definizione di res publi$c a$, discussa e strutturata da Cicerone in una consapevole costruzione giuridico-filosofica volutamente rappresentata come propriamente romana (ossia, non da doctores greci); il che forse spiega anche l'assenza di uomini della levatura di Polibio e Panezio. ${ }^{22}$

Strutturalmente la costruzione della frase si presenta nella forma del sillogismo, come di regola rappresentato con due proposizioni legate da autem. La prima enuncia la celebre definizione di res publica: «Est igitur ... res publica res populi.» Qui l'aggettivo (publica) appare legato, mediante un genitivo relazionale o di appartenenza, al sostantivo populus a significare l'identificazione della res publica con questo. Cosa debba intendersi per populus Cicerone/Scipione lo precisa immediatamente nelle due sottoproposizioni che seguono.

Il dato che qui interessa è che, in questo caso, la nozione di res populi è configurata in un senso giuridico-istituzionale, piuttosto che economico-materialistico. A parte l'uso analogo di res populi come res publica già chiaro in Plauto (Poen. 524: praesertim in re populi placida atque interfectis hostibus non decet tumultuari) e Persio (4, 1: «Rem populi tractas?») (di età neroniana), non osta in questa prospettiva l'indicazione del Pohlenz per cui, in base a un noto riferimento di Festo a Catone, l'espressione res publica in senso giuridico-istituzionale non sarebbe anteriore al II secolo a.C.:23

Fest. sv. Struere (L. 408, 31): antiqui dicebant pro adicere, augere. Unde industrios quoque M. Cato (or. inc. 19): "Iure, lege, libertate, republica, communiter uti oportet: gloria atque honore, quomodo sibi quisque struxit; aut in XII $(1,2)$ quod est: «si calvitur pedemve struit».

Pur con riserva di tutte le cautele del caso, direi allora che in questo luogo del De re publica, l'idea di una libera res publica giuridicamente legittimata appaia già ipostatizzata in un senso "costituzionale" avvicinabile a quello moderno di "Stato"; la parola populus è qui ormai un'astrazione, a indicare, nell'espressione res publica, un'idea di *populitas o *populium sconosciuta al latino arcaico; ${ }^{24} \mathrm{e}$, mi permetto di aggiungere, forse sovrapponendosi a un significato originario di populus come "esercito in armi". ${ }^{25}$

La possibilità che la nozione di res publica propugnata dai partecipanti del circolo scipionico s'identificasse esclusivamente col suo territorio va quindi respinta; ${ }^{26}$ e mi sembra dirimente in tal senso anche quanto afferma lo stesso Cicerone in un passo noto dell'epistolario ad Attico, parlando della fuga di Pompeo dall'Italia:

Cic. ad Att. 7, 11, 3: «non est» inquit, «in parietibus res publica». At in aris et focis.

22 Su questa celebre e studiatissima testimonianza si v. CANCELLI, Introduzione, pp. 61-76, ma anche CASCIONE, C. Consensus. Problemi di origine, tutela processuale, prospettive sistematiche. Napoli: Editoriale Scientifica, 2003, pp. 67-70.

23 CANCELLI, Introduzione, 64 e passim. Per il riferimento cronologico v. ora POHLENZ, M. La Stoa. Storia di un movimento spirituale (1959). Milano: Rusconi, 2005, pp. 1-1036, in part. p. 537.

24 Analogamente Ovidio usa res regia in sostituzione di regnum: Ovid.fast. 6, 595: regia res scelus est; Ovid. ex Pont. 2, 9, 11: Regia, crede mihi, res est succurrere lapsis conuenit et tanto quantus es ipse uiro. Dunque res populi come res regni. Ancora, per tutto, CANCELLI, Introduzione, p. 64.

25 Ne discuto in SACCHI, O. La nozione di ager publicus populi Romani nella lex agraria del 111 a.C. come espressione dell'ideologia del suo tempo. Tijdschrift voor Rechtsgeschiedenis, 2005, 73, pp. 19-42, spec. p. 24 e passim.

26 Per lo status quaestionis v. CANCELLI, Introduzione, pp. 74-76 e passim. 


\section{Il concetto di populus}

La seconda parte dell'enunciato di Scipione riguarda invece, come è altrettanto noto, la definizione di populus e si articola a sua volta in altre due sottoproposizioni affermative, di cui la prima in forma negativa (non omnis hominum coetus quoquo modo congregatus); mentre la seconda in forma affermativa (sed coetus multitudinis iuris consensu et utilitatis communione sociatus). Qui populus, come detto, non è più l'esercito, ovvero qualsiasi aggregato che si mette insieme in una qualche maniera, ma una moltitudine sostenuta dall'"armonia del diritto", quindi giuridicamente qualificata (iuris consensu) e da un "utile comune" (utilitatis communione sociatus). In questa prospettiva, ciò che spingerebbe gli uomini ad aggregarsi in società non sarebbe, dunque, come in Polibio, la coscienza di una debolezza (eius autem prima causa coeundi est non tam inbecillitas); quanto un istinto naturale (quam naturalis quaedam hominum quasi congregatio). ${ }^{27}$

Così dottrina attenta (in base anche a de re p. 3, 31, 43 dove Scipione legge in negativo il nesso res publica/res populi ${ }^{28}$ ) ha individuato nel vinculum iuris, consensus e coetus sociatus gli elementi fondanti della nozione di populus, tenuti insieme dall'utilità comune ed escludendo la presenza di un tiranno (ubi tyrannus est, [...] plane nullam esse rem publicam):

Cic. de re p. 3, 31, 43: (Scipio) reportare. ergo illam rem populi, id est rem publicam, quis diceret tum dum crudelitate unius oppressi essent universi, neque esset unum vinculum iuris nec consensus ac societas coetus, quod est populus? atque hoc idem Syracusis. urbs illa praeclara, quam ait Timaeus Graecarum maxumam, omnium autem esse pulcherrimam, arx visenda, portus usque in sinus oppidi et ad urbis crepidines infusi, viae latae, porticus, templa, muri nihilo magis efficiebant, Dionysio tenente ut esset illa res publica; nihil enim populi, et unius erat populus ipse. ergo ubi tyrannus est, ibi non vitiosam, ut heri dicebam, sed, ut nunc ratio cogit, dicendum est plane nullam esse rem publicam.

\section{La nozione di proprietà in de off. 1, 21 e nella dottrina politica di Panezio}

È inutile che mi dilunghi su questo tema molto indagato, ci si può chiedere tuttavia quale fosse l'utile concreto di questa spinta all'aggregazione sociale che si voleva così giuridicamente disciplinata. La risposta per me è data dal tenore di alcuni passaggi del De officiis tra i quali spiccano le due testimonianze prima citate:

Cic. de off. 1, 20-21: Sed iustitiae primum munus est, ut ne cui quis noceat, nisi lacessitus iniuria, deinde ut communibus pro communibus utatur, privatis ut suis. Sunt autem privata nulla natura, sed aut vetere occupatione, ut qui quondam in vacua venerunt, aut victoria, ut qui bello potiti sunt, aut lege, pactione, condicione, sorte; ex quo fit, ut ager Arpinas Arpinatium dicatur, Tusculanus Tusculanorum; similisque est privatarum possessionum discriptio. Ex quo, quia suum cuiusque fit eorum, quae natura fuerant communia, quod cuique optigit, id quisque teneat; e quo si quis [quaevis] sibi appetet, violabit ius humanae societatis.

\footnotetext{
27 Polyb. 6, 5, 7: «allora dunque, dopo che, come gli altri animali, anche loro si sono radunati insieme (ed è verosimile che anch'essi si raggruppino con gli individui della stessa specie, a causa della loro naturale debolezza), è ineluttabile che colui che spicca per forza fisica e audacia di spirito guidi e comandi» [trad. it. di G. Colesanti in NICOLAI, R. (a cura di). POLIBIO. Storie. Vol. 2. Roma: Newton Compton, 1998, p. 283]. CASCIONE, op. cit., p. 68.
} 
Cic. de off. 2, 73: Hanc enim ob causam maxime, ut sua tenerentur, res publicae civitatesque constitutae sunt. Nam, etsi duce natura congregabantur homines, tamen spe custodiae rerum suarum urbium praesidia quaerebant.

Rivediamole ancora più da vicino. Il primo testo (de off. 1,20) esordisce con la seguente considerazione: il cittadino che vive secondo giustizia rispetterà gli altri usando le cose comuni della res publica in quanto comuni (deinde ut communibus pro communibus utatur); e userà come sue solo le cose che gli appartengono (privatis ut suis). Come non vedere qui un richiamo a G. Lelio Sapiente ricordato nel De re publica, per bocca di Manilio, come colui che, oltre a distinguersi per l'eccellenza della sua ars iuris, si era interrogato sull'applicabilità al diritto romano della distinzione tra ciò che è "proprio" e ciò che è di "altri"?:

Cic. de re p. 1, 13, 20: (Laelius) immo vero te audiamus, nisi forte Manilius interdictum aliquod inter duos soles putat esse componendum, ut ita caelum possideant ut uterque possederit» tum Manilius: "pergisne eam, Laeli, artem inludere, in qua primum excellis ipse, deinde sine qua scire nemo potest quid sit suum quid alienum?

Il riferimento alla questione agraria dell'epoca graccana mi pare quindi in questo caso evidente. Anche tenendo conto di quanto dice Plutarco sullo stesso Lelio che, come detto, nel 145, suo anno di carica pretorile, avrebbe proposto una legge agraria (secondo Plutarco) di contenuto analogo a quella che Tiberio Gracco avrebbe presentato più tardi:

Plut. Tib. Gracc. 8, 5: «Già Gaio Lelio, l'amico di Scipione, aveva tentato di sanare questa situazione; ma poi, di fronte opposizione dei potenti e per timore di torbidi, aveva desistito. Appunto per questa mossa era stato soprannominato 'Saggio' o 'Prudente' (in latino il termine sapiens sembra avere entrambi i significati). Tiberio, eletto tribuno subito decise di accingersi lui stesso all'impresa. E secondo i più lo fece per incitamento del retore Diofane e del filosofo Blossio». ${ }^{29}$

L'idea si rafforza pensando alla raffinata allusione sull'apparizione nel cielo di Roma di "due soli" (di cui si diceva prima) che avrebbero "posseduto il cielo". Il riferimento è al parelio, un fenomeno ottico/atmosferico (avvenuto appunto nel 129 a.C.), dovuto alla rifrazione prodotta nel cielo da cristalli di ghiaccio che i romani consideravano annunzio di sventura; ${ }^{30}$ e che, nel caso di specie, interpretarono come sinistro presagio delle agitazioni per la legge Sempronia agraria e della morte di Scipione. Secondo Cicerone, il "vero" Sole sarebbe stato l'Emiliano; ${ }^{31}$ mentre Tiberio solo una sua falsa geminazione. ${ }^{32}$

E non è tutto perché, più avanti, nel De officiis in 2, 73, nella prima parte del testo, troviamo una vera e propria invettiva contro ogni proposito di distribuzione equitativa di terra, usandosi come pretesto il tentativo di riforma agraria tentato dal tribuno (L. Marcio) Filippo nel 104 a.C. che, sul presupposto che «non c'erano in città più di duemila persone che avevano una proprietà (qui rem haberent)», si spese in una serie di discorsi demagogici giudicati da Cicerone addirittura pericolosi:

Cic. de off. 2, 73: In primis autem videndum erit ei, qui rem publicam administrabit, ut suum quisque teneat neque de bonis privatorum publice deminutio fiat. Perniciose enim Philippus in tribunatu cum legem agrariam ferret, quam tamen antiquari facile passus est

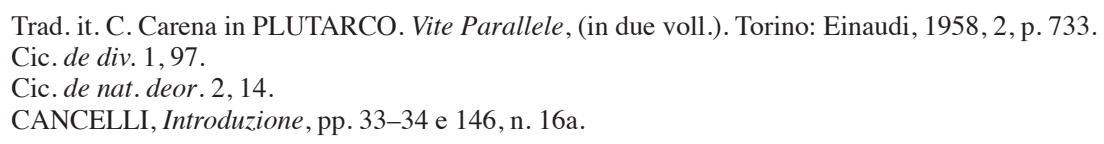


et in eo vehementer se moderatum praebuit; sed cum in agendo multa populariter, tum illud male, «non esse in civitate duo milia hominum, qui rem haberent». Capitalis oratio est ad aequationem bonorum pertinens, qua peste quae potest esse maior? Hanc enim ob causam maxime, ut sua tenerentur, res publicae civitatesque constitutae sunt. Nam, etsi duce natura congregabantur homines, tamen spe custodiae rerum suarum urbium praesidia quaerebant.

Sembra anche qui una rievocazione di Lelio che, come detto, si guadagnò l'appellativo di "sapiente" o "prudente", proprio per non aver insistito in una legge di riforma agraria invisa ai conservatori; o di Scipione Emiliano che si prodigò (come ripeto ancora), forse a caro prezzo, per salvare gli italici dalle commissioni triumvirali graccane intenzionate a espropriare i possedimenti garantiti da foedera. Cicerone è molto esplicito: le res publicae e le civitates sono costituite proprio per consentire a ciascuno di conservare quello gli appartiene (Hanc enim ob causam maxime, ut sua tenerentur, res publicae civitatesque constitutae sunt).

Ritorniamo allora a Panezio che in de off. 1, 21, prendendo atto (o forse per legittimare) la recente predazione di terra perpetrata dai nuovi padroni del mondo (ricordo ancora che l'Emiliano fu il distruttore di Cartagine), propose una teoria politica che riconobbe alla res publica (quindi allo "Stato") e alla "proprietà" (l'espressione usata da Cicerone è privatarum possessionum come già nel 60 a.C. in una nota epistula ad Attico ${ }^{33}$ ) una stessa origine. Come già detto: la prima, ossia la res publica, sarebbe nata per proteggere la seconda; cioè per difendere le "cose di ciascuno", secondo un andamento delle cose ritenuto "naturale" (de off. 1, 21: quae natura fuerant communia, quod cuique optigit, id quisque teneat $\left.{ }^{34}\right)$.

Da notare la sottigliezza: qui Panezio, o forse più Cicerone, cerca di superare il problema della "innaturalità" del concetto di appartenenza o proprietà sostenuto da Crisippo (v. sempre de off. 1, 21: sunt autem privata nulla natura), tanto più se procurato in modo predatorio, giocando sull'idea della "naturale" propensione degli uomini ad aggregarsi in società (questa volta de off. 2,73: etsi duce natura congregabantur homines) per la difesa

33 Cic. ad Att. 1, 19, 4: Ego autem magna cum agrariorum gratia confirmabam omnium privatorum possessiones. Cicerone qui, come in altri luoghi dell'epistolario (come ad esempio ad Att. 1, 18, 6), fa riferimento all'annosa questione della redistribuzione delle terre demaniali (ager publicus) oggetto di appropriazione latifondistica a favore di privati (coloni espropriati) o di veterani dell'esercito congedati (come al tempo di Silla) che risaliva al tempo dei Gracchi. Nel caso di specie Cicerone (che si era opposto, quando console, alla proposta di Servilio Rullo) parla della rogatio del tribuno della plebe Flavio (dietro il quale stava Pompeo) volta a favorire i veterani delle guerre d'Asia.

34 Il senso delle parole id quisque teneat è di "possideat tanquam suum" già in M. T. Ciceronis de Officiis libri tres ad modum J. Minellii illustrati. Neapoli: Franciscus Masi Typocosmetor, 1825, p. 26. Il senso giuridico di tale forma di appartenenza (ager privatus e/o ager datus adsignatus) si esplicita ex post in una nota testimonianza di Ulpiano (D. 45, 1, 38, 8 Ulp. 49 ad Sab.), dove (a proposito di uno schiavo) è chiaramente enunciato il principio per cui: nessuno dubita che si possa "tenere", là dove non sia possibile "possedere" per il ius civile (licet enim possidere civiliter non possint, tenere tamen eos nemo dubitat). Utilizza tale fonte per chiarire il significato dei termini habere e possidere della legge agraria del 111 a.C., CHOUQUER, op. cit., p. 217. Ciò in virtù della duplice accezione giuridica del termine habere che designava il possesso del dominus o la semplice detenzione per conto terzi: D. 45, 1, 38, 9 (Ulp. 49 ad Sab.): "habere" dupliciter accipitur: nam et eum habere dicimus, qui rei dominus est et eum, qui dominus quidem non est, sed tenet: denique habere rem apud nos depositam solemus dicere. Se è molto probabile che questo fosse il significato giuridico delle parole habere possidere della lex del 111, la testimonianza del De officiis di Cicerone dimostra che ciò valesse anche per l'età cesariana. 
personale, ma soprattutto dei propri beni comunque acquisiti (ancora in de off. 2, 73: tamen spe custodiae rerum suarum urbium praesidia quaerebant). ${ }^{35}$

A cosa allude, Cicerone lo precisa con una breve tassonomia che per me riassume le vicende che segnarono in generale la storia del passaggio dalla possessio dell'ager publicus al dominium quiritario. Basta una rapida rilettura del passo in questione per rendersi subito conto che il retore abbia fornito, forse involontariamente, anche un elenco delle varie tipologie di appartenenza che scandirono la storia dell'affermazione della proprietà fondiaria nel diritto romano repubblicano e che costituirono anche le varie tipologie di appartenenza in gioco nella questione agraria dell'epoca graccana: (de off. 1, 21): sed aut vetere occupatione, ut qui quondam in vacua venerunt, aut victoria, ut qui bello potiti sunt, aut lege, pactione, condicione, sorte. Come si vede, il retore, forse anche aggiungendo qualcosa al discorso di Panezio, menziona: 1) la vetus occupatio, ossia la terra posseduta dai prischi latini, detti da Ennio con parola osca Casci; ${ }^{36}$ poi l'ager che, diventato vacuus (non posseduto da alcuno), il retore chiama occupatorius, ossia quello acquisito in virtù di predazione bellica, dunque molto probabilmente quello di originaria pertinenza del populus come esercito (vetere occupatione, ut qui quondam in vacua venerunt, aut victoria, ut qui bello potiti sunt); ${ }^{37}$ 2) la legge (a cominciare dagli agri dati adsignati con le prime colonie), categoria di cui emblematica è la lex agraria del 111 a.C., dove l'incertezza terminologica della doppia endiadi ager poplicus populi Romani denuncia la persistenza di una fase di assestamento, con la netta contestuale affermazione dell'ager privatus ancora qualificato come titolarità di un uti frui habere possidere e quindi non ancora dominium; ${ }^{38}$ 3) i terreni acquisiti mediante pactio, ossia l'attività negoziale privata o pubblica come, ad esempio, la mancipatio dei praedia tam urbana quam rustica di Gai 1, 120; oppure l'ager privatus vectigalisque o quaestorius della legge del 111 a.C.;9 ovvero, l'ager publicus confermato (11. 36-39) o l'ager publicus fruendus datus e l'ager publicus in trientabuleis fruendus datus (11. 31-32); ${ }^{40} 4$ ) i terreni acquisiti mediante condicio, ossia per trattato (si pensi alla terra che Publio Cornelio Scipione Maggiore acquisì al popolo romano e che fu concessa al re Iempsale in virtù di patto $($ foedus $) ;{ }^{41}$ ovvero ai possedimenti privati italici il cui possesso era garantito da foedera per cui, come detto, a favore dei quali intervenne

35 Lo stoico Crisippo risolveva il problema della inesistenza in natura della proprietà (ecco il sunt autem privata nulla natura in de off. 1,21) usando la metafora del teatro, dove lo spettatore chiama suo il posto che occupa e si considera, questa, una cosa legittima. Si superava così il problema di qualificare come "proprio" qualcosa che nel mondo invece si sentiva come comune a tutti: Cic. de fin. 3, 20, 67: Sed quem ad modum, theatrum cum commune sit, recte tamen dici potest eius esse eum locum quem quisque occuparit, sic in urbe mundove communi non adversatur ius quo minus suum quidque cuiusque sit.

36 Cfr. Enn.ann. V 24: Quam Prisci casci populi tenuere Latini; Cic. Tusc. 1, 27: Itaque unum illud erat insitum priscis illis, quos cascos appellat Ennius, esse in morte sensum neque excessu vitae sic deleri hominem, ut funditus interiret; idque cum multis aliis rebus, tum e pontificio iure et e caerimoniis sepulcrorum intellegi licet (...); Varro l.L. 7, 28: primum cascum significat vetus; secundo eius origo Sabina, quae usque radices in Oscam linguam egit. Cascum vetus esse significat Ennius quod ait: «Quam Prisci casci populi tenuere Latini».

37 Sulle tipologie storiche dell'ager occupatorius v. ora CHOUQUER, op. cit., p. 66.

38 SACCHI, La nozione di ager publicus populi Romani nella lex agraria del 111 a.C., p. 23 ss.

39 Linee 49, 58 e 66. Sull'ager privatus vectigalisque v. ora CHOUQUER, op. cit., p. 197 ss. e passim. Sulle vendite questorie v. anche SACCHI, O. Limiti geografici, cenni di storia ed organizzazione dell'ager Campanus fino alla deditio del 211 a.C. In: FRANCIOSI, G. (a cura di). La romanizzazione della Campania antica. Napoli: Jovene, 2002, pp. 19-86, spec. p. 83 e passim.

40 CHOUQUER, op. cit., p. 62 e passim.

41 Cic. de lege agr. 2, 22, 58. 
proprio Scipione Emiliano nel 129 a.C.);42 5) i terreni acquisiti sorte, ossia per sorteggio, come fece Silla e nei casi previsti dalla legge del 111 a.C. ${ }^{43}$ Rilevante storicamente, per tale categoria, la disciplina del modus agri alienabile mediante mancipatio, ossia il lotto di terreno assegnato ai reduci nella deduzione colonaria consistente nell'assegnazione di una quota ideale della centuria con sorteggio. ${ }^{44}$

Sono queste le categorie di terreno rientranti nella più ampia categoria dell'ager publicus che nel corso del tempo diventarono suscettibili di appropriazione privata sovrapponendosi anche alla tradizionale classificazione dell'ager augurale di cui come detto dice Varrone in l.L. 5, 5, 33 (Romanus, Gabinus, peregrinus, hosticus, incertus). Cicerone, con la frase ex quo fit, ut ager Arpinas Arpinatium dicatur, Tusculanus Tusculanorum, è a mio avviso (forse inconsapevolmente) testimone di una significativa differenza di approccio da cui possono dedursi implicitamente due principi importanti: anzitutto che la res publica (e dunque per chiunque pretendesse di agire in suo nome) non potesse essere giuridicamente considerata "proprietaria" delle res publicae (ciò in conformità ai principi di un diritto romano ormai laicizzato: ager publicus=ager incertus?); ${ }^{45}$ in secondo luogo, che tale possibilità dovesse invece riconoscersi per i singoli cittadini. Premettendo ciò, si dovrebbe parlare allora in senso giuridico per l'ager publicus solo di mera "appartenenza" (pur suscettibile di adsignatio); 46 mentre di “proprietà" solo per l'ager privatus. Quest'ultimo, pur configurato sempre come una forma di possesso, recava in sé già l'idea di proprietà in virtù del principio per cui "dove è possibile la proprietà è possibile anche il possesso"; 47 diversamente, l'ager publicus, mancando capacità proprietaria soggettiva o oggettiva in capo alla res publica, dovrebbe essere configurato solo come mera appartenenza, in virtù del principio per cui "dove non è possibile la proprietà non è possibile neanche il possesso". ${ }^{8}$ Chissà che con l'espressione ager incertus la dottrina augurale non intendesse fare

42 L'ager sorti datus adsignatus, assegnato a cittadini romani viritim o nella costituzione di colonie a cui si garantì il possesso ereditario e l'iscrizione al censo (11. 2-3; 7-11; 15-16; 19-20); 1'ager sorti colendi causa, consistente in assegnazioni del surplus recuperato da possessori precedenti, in regime di esenzione da vectigal qualificato possidere, habere o convertito in ager privatus (11. 13-14; 19-20). Cfr. ora per tutto CHOUQUER, op. cit., p. 62 e passim.

43 Liv. epit. 89; Lex agraria 11. 2-3, 7-11, 15-16, 19-20 (ager sorti datus assignatus); 11. 13-14, 19-20 (ager sorti colendi causa).

44 Cfr. SACCHI, Ager est non terra, p. 178, n. 45; CHOUQUER, op. cit., p. 97 s. e passim.

45 Si v. per questo JHERING, R. Sul fondamento della protezione del possesso. Trad. it. F. Forlani. Milano: Francesco Vallardi, 1872, pp. 109-123, spec. pp. 115-119.

46 JHERING, op. cit., p. 115 così chiarisce tale concetto: «Io parto dal principio, che le nozioni della «appartenenza» giuridica "alicujus esse" e della proprietà, non si fondono assieme, che in quella vece qualche cosa mi può "appartenere", senza essere in mia proprietà, vale a dire senza soggiacere ai principii che sono enunciati pella stessa, rispetto all'acquisto, perdita, protezione, comproprietà e tocca via. I miei capelli mi "appartengono" e singole treccie di capelli tagliati sono oggetto di commercio e di proprietà, ma sino a che esse aderiscono al capo non vi trovano applicazione le nozioni del possesso e della proprietà». Come si legge in tema di lex Aquilia in D. 9, 2, 13pr. (Ulp. 18 ad ed.): dominus membrorum suorum nemo videtur.

47 JHERING, op. cit., p. 119.

48 Cfr. l'ampia dimostrazione di JHERING, op. cit., pp. 109-119 che fra l'altro ricorda che i giuristi romani quando parlano del rapporto tra lo "Stato" e le res publicae non usano mai il termine dominium, ma la più indeterminata espressione ius civitatis: D. 43, 8,2, 2 (Ulp. 68 ad ed.): (...) loca enim publica utique privatorum usibus deserviunt, iure scilicet civitatis, non quasi propria cuiusque (...). La ratio di questo particolare stato giuridico delle res publicae risiederebbe nell'essere queste destinate a servire i cittadini (usus publicus), non la res publica come persona giuridica (JHERING, op. cit., ivi p. 116). L'applicabilità della nozione di proprietà alle res publicae può essere quindi negata in diritto romano perché: a) vengono contrapposte al vero patrimonio dello Stato (patrimonium, pecunia, bona populi, fisci, ecc.): D. 18, 1, 6pr.; 
riferimento proprio a questo, dato che la destinazione delle res publicae non era di servire allo "Stato", ma ai cittadini (usus publicus).

Lascio però aperta questa complessa questione, con riserva di ritornarvi in altra sede.

\section{Il riferimento alla iuris discriptio (o distributio) in Cic. de off. 1, 21}

L'ultima considerazione è per l'inciso similisque est privatarum possessionum discriptio. La iuris discriptio (o distributio ${ }^{49}$ ) a cui fa riferimento Cicerone in chiusura del frammento di cui in de off. 1, 21, nel lessico giuridico-istituzionale romano era l'azione del legislatore volta a regolamentare i regimi dell'appartenenza fondiaria con indiretta, ma chiara, allusione all'ordinamento centuriato (e qui il Vico avrebbe molto da dire) ${ }^{50} \mathrm{Si}$ diceva all'inizio della possibilità che il dibattito (e quindi la configurazione) giuridico-istituzionale della nozione di res publica in rapporto all'idea stessa di proprietà, sviluppatosi nel circolo scipionico e perfezionatosi con Cicerone, sia potuto diventare un vero paradigma per i tempi a venire; e di come questo possa essere stato molto influenzato dalla questione agraria del periodo graccano.

A ulteriore conferma di ciò si può forse addurre un altro elemento: la posizione assunta nel circolo scipionico dal partito conservatore che può aver avuto come ideale e significativo punto di riferimento proprio l'ordinamento centuriato. Il consensus e il viculum iuris (insieme al coetus sociatus) - su cui questi intellettuali fondarono la ragione d'essere della res publica (e quindi della stessa fonte legittimante della proprietà dei beni di ogni cittadino) - non potevano prescindere da una iuris distributio o discriptio considerata come l'azione del legislatore diretta a formarla. L'indicazione è inequivocabile nelle seguenti testimonianze:

Cic. de re p. 2, 22, 39-40: [39] (Scipio) duodeviginti censu maximo. deinde equitum magno numero ex omni populi summa separato, relicuum populum distribuit in quinque classis, senioresque a iunioribus divisit, easque ita disparavit ut suffragia non in multitudinis sed in locupletium potestate essent, curavitque, quod semper in re publica tenendum est, ne plurimum valeant plurimi. quae discriptio si esset ignota vobis, explicaretur a me; nunc rationem videtis esse talem, ut equitum centuriae cum sex suffragiis et prima

$18,1,72,1 ; 41,1,14$ pr.; 43, 8, 2, 4; 50, 16, 17pr. La qual cosa implica che quanto scritto in D. 1, 8, 1pr. (res publicae nullius in bonis esse creduntur) e in D. 1, 8, 6, 2 (et sacrae et religiosae et sanctae nullius in bonis sunt), ma ancora in Gai 2, 11: Quae publicae sunt, nullius uidentur in bonis esse; ipsius enim uniuersitatis esse creduntur, significa negazione di proprietà privata (ivi, 117, n. 2); b) i giuristi negavano la possibilità che su queste potessero riconoscersi le prerogative tipiche del proprietario (usucapione, servitù, compera, stipulazione, ecc.): D. 45, 1, 83, 5; 18, 1, 34, 1; 8, 2, 1pr.; 41, 3, 9; 43, 11, 2 (ivi, 118, n. 1).

49 Così CANCELLI, Introduzione, p. 69.

50 Così ancora CANCELLI, Introduzione, pp. 68-70 in base a: Cic. pro Quinct. 14: Quis tandem nobis ista iura tam aequa discribit?; pro Caec. 74: quid, inquam, prodest fundum habere, si, quae diligentissime descripta a maioribus iura finium, possessionum, aquarum itinerumque sunt, haec perturbari aliqua ratione commutarique possunt?; Verr. 2, 5, 27: praetio, non aequitate, iura descripserat; pro Sextio 91: homines nondum neque naturali neque civili iure descripto fusi per agros ac dispersi vagarentur; de orat. 1, 33: quae vis alia potuit aut dispersos homines unum in locum congregare aut a fera agrestique vita ad hunc humanum cultum civilemque iura describere?; de re p . 1, 2: a quibus civitatibus iura discripta sunt; de leg. 1, 17: haec tractanda, quae conposita sunt et descripta iura et iussa populorum; de leg. 2, 11: Quae sunt autem varie et ad tempus descriptae populis, [...] eos qui perniciosa et iniusta populis iussa descripserint; de off. 2, 15: Urbes vero sine hominum coetu non potuissent nec aedificari nec frequentari, ex quo leges moresque constituti, tum iuris aequa discriptio certaque vivendi disciplina. In: M. T. Ciceronis de Officiis libri tres ad modum J. Minellii illustrati, p. 26, il termine descriptio è già sciolto come definitio et divisio. 
classis, addita centuria quae ad summum usum urbis fabris tignariis est data, LXXXVIIII centurias habeat; quibus e centum quattuor centuriis (tot enim reliquae sunt) octo solae si accesserunt, confecta est vis populi universa, reliquaque multo maior multitudo sex et nonaginta centuriarum neque excluderetur suffragiis, ne superbum esset, nec valeret nimis, ne esset periculosum. [40] in quo etiam verbis ac nominibus ipsis fuit diligens; qui cum locupletis assiduos appellasset ab asse dando, eos qui aut non plus mille quingentos aeris aut omnino nihil in suum censum praeter caput attulissent, [...] ita nec prohibebatur quisquam iure suffragii, et is valebat in suffragio plurimum, cuius plurimum intererat esse in optimo statu civitatem.

Cic. de re p. 4, 2, 2: (2) Scipio: "gratiam, quam commode ordines discripti aetates classes equitatus, in quo suffragia sunt etiam senatus, nimis multis iam stulte hanc utilitatem tolli cupientibus, qui novam largitionem quaerunt aliquo plebiscito reddendorum equorum $[\ldots]$...

Qui è sempre Scipione che parla e la discriptio, ovvero l'ordinamento centuriato serviano, potrebbe anche considerarsi l'espressione concreta (una sorta di ipostasi) di quel consensus su cui si formava il vinculum iuris che, in questa nuova concezione di res publica, doveva sostanziarsi nell'adesione a una costruzione armonica del ius. Si voleva, in altre parole, che i compiti e il ruolo di ciascun cives, fossero inquadrati in un ordine gerarchico di cui l'ordinamento serviano fu considerato momento genetico ed espressione emblematica. Del ben noto testo del De re publica (2, 22, 39-40), dove Cicerone descrive il meccanismo serviano, evidenzio qui soprattutto la parte finale: "non si vietava a nessuno il diritto di voto, ma nel voto valeva di più, colui che aveva maggiore interesse che la comunità politica fosse in optimo statu civitatem". Dunque gli appartenenti al partito conservatore o gli ottimati.

Tutt'altra cosa quindi rispetto all'aequabilitas e societas iuris del partito democratico che, all'opposto, perseguiva l'ideale di una giustizia distributiva senza alcuna distinzione di sorta. Non per caso Livio avrebbe affermato che, con l'ordinamento centuriato di Servio, si sarebbero manifestate le differenze dei gradus dignitatis:

Liv. 1, 42, 4: Seruium conditorem omnis in civitate discriminis ordinumque quibus inter gradus dignitatis fortunaeque aliquid interlucet posteri fama ferrent. Censum enim instituit, rem saluberrimam tanto futuro imperio, ex quo belli pacisque munia non viritim, ut ante, sed pro habitu pecuniarum fierent; tum classes centuriasque et hunc ordinem ex censu discripsit, vel paci decorum vel bello.

In questo quadro direi che acquisti maggiore significato il noto frammento del De officiis che, insieme a De re p. 1, 25, 39 e de off. 2, 73, ha costituito prevalentemente l'oggetto di questo mio intervento. La frase similisque est privatarum possessionum discriptio in de off. 1, 21 non può che essere un riferimento (in ottica conservatrice) al regime fondiario esistente che sin dalle riforme dei Gracchi i romani tentarono invano di razionalizzare. 